\title{
Compressed Amplatzer Vascular Plug II Embolization of the Left Subclavian Artery for Thoracic Endovascular Aortic Repair is Efficient and Safety Method Comparable to Conventional Coil Embolization
}

\author{
Kensuke Matsumoto,* Yasufumi Ohuchi,* Shinsaku Yata,* Akira Adachi,* Masayuki Endo,* Shohei Takasugi,* \\ Shinya Fujii,* Masayuki Hashimoto, $\dagger$ Toshio Kaminou, $\ddagger$ Toshihide Ogawa, $\S$ Yoshikazu Fujiwara,ll Munehiro \\ Saiki J and Motonobu Nishimurall \\ *Division of Radiology, Department of Pathophysiological and Therapeutic Science, School of Medicine, Tottori University Faculty of \\ Medicine, Yonago 683-8504, Japan, †Department of Radiology, Tottori Municipal Hospital, Tottori 680-8501, Japan, $\$$ Department of \\ Radiology, Osaka Minami Medical Center, Kawachinagano 586-8521, Japan, \$Department of Radiology, Kurashiki Heisei Hospital, \\ Kurashiki 710-0826, Japan, IIDivision of Organ Regeneration Surgery, Department of Surgery, School of Medicine, Tottori University \\ Faculty of Medicine, Yonago 683-8504, Japan, and Department of Cardiovascular surgery, Hiroshima City Hiroshima Citizens Hospital, \\ Hiroshima 730-8518, Japan
}

\begin{abstract}
Background Left subclavian artery (LSA) embolization is occasionally required to prevent type II endoleak in the thoracic endovascular aortic repair (TEVAR) procedure. This is a retrospective study comparing compressed Amplatzer Vascular Plug II embolization (CAE) and conventional coil embolization (CCE) in preventing retrograde flow into the aneurysmal sac through the LSA after TEVAR.
\end{abstract}

Methods We retrospectively reviewed the records of patients who underwent CAE or CCE of the LSA during TEVAR from June 2013 to March 2016 in our hospital. The efficacy, safety and cost of each method were compared between two groups.

Results Thirty patients underwent LSA embolization during TEVAR. Six CCEs in 6 patients were performed from June 2013 to November 2013, while twenty-four CAEs in 24 patients were performed from December 2013 to March 2016. Technical success was achieved in all patients in both groups. No embolization-related complications or type II endoleaks from LSA were recorded during the follow-up period in all patients. In both groups, all embolic materials were detected in the proximal portion of the LSA from the LSA orifice to the vertebral artery origin and no vertebral artery occlusions were detected. The mean compression ratio of AVP II was $58 \pm 5.9 \%$ of predicted length of standard procedure. In the CAE group, one AVP II was sufficient to achieve complete LSA occlusion in all patients. On the other hand, multiple coils $(10.2 \pm 2.7)$ were used in the

\footnotetext{
Corresponding author: Kensuke Matsumoto, MD matsumoto-k@v103.vaio.ne.jp

Received 2018 November 14

Accepted 2018 December 17

Abbreviations: AVP II, Amplatzer Vascular Plug II; CAE, compressed Amplatzer Vascular Plug II embolization; CCE, conventional coil embolization; CT, computed tomography; LSA, left subclavian artery; TEVAR, thoracic endovascular aortic repair
}

CCE group $(P<.01)$, resulting in a significantly lower cost incurred in the CAE group (CAE: 129,000 JPY vs. CCE: $639,600 \pm 140,060 \mathrm{JPY} ; P<.01)$.

Conclusion The CAE is a useful and cost-effective procedure for TEVAR-related LSA embolization.

Key words endovascular procedures; aortic aneurysm; subclavian artery; embolization

In order to establish the requisite landing zone for thoracic endovascular aortic repair (TEVAR), it is often necessary to cover the orifice of the left subclavian artery (LSA) with an endovascular graft. This procedure has been performed in up to $68.0 \%$ of repairs. ${ }^{1-4}$ In such situations, retrograde flow through the LSA may cause a type II endoleak, aneurysmal sac expansion or rupture. ${ }^{1,5-9}$ As such, LSA occlusion is a procedure that is widely established to be necessary in TEVAR.

Embolization of the proximal portion of the LSA from the LSA orifice to the vertebral artery origin has been traditionally performed by conventional coil embolization (CCE). CCE, while often successful, can occasionally be problematic because of coil misplacement and the need for multiple coils (Fig. 1). ${ }^{9}$ In the last decade, Amplatzer Vascular Plug II (AVP II) (St. Jude medical, St. Paul, MN) has been commercially available in Japan. AVP II has three multi-layered nitinol mesh lobes for rapid embolization of the vessel and has since been used for embolization of the internal iliac artery during endovascular aortic repair for abdominal aortic aneurysms..$^{10}$ The effectiveness of AVP II embolization for the LSA in selected cases has also been reported in the literature. ${ }^{5,11,12}$ However, AVP II application for the LSA is technically difficult because the proximal portion of the LSA from the LSA orifice to the vertebral artery origin is not long enough for the AVP II place- 

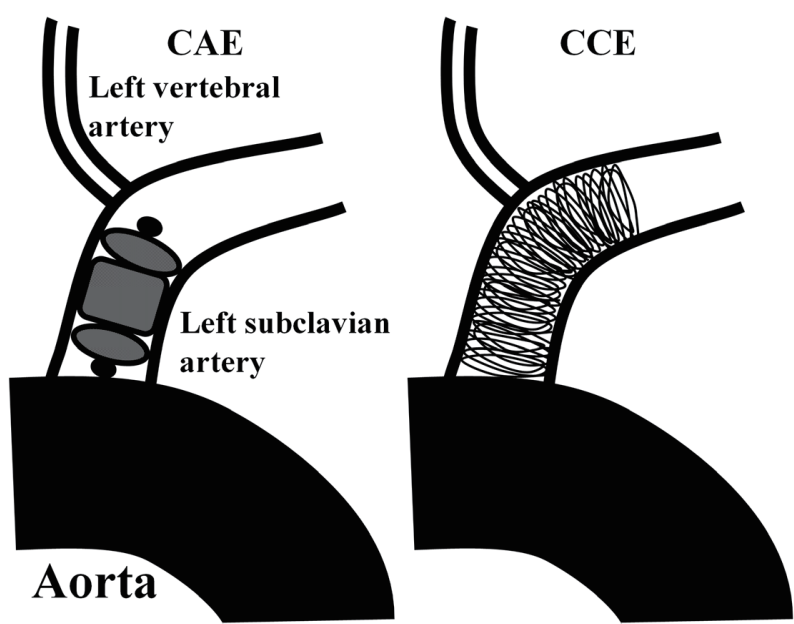

Fig. 1. The schema of appropriate AVP II placement by CAE and potential inappropriate coil placement by CCE method. AVP II, Amplatzer Vascular Plug II; CAE, compressed Amplatzer Vascular Plug II embolization; CCE, conventional coil embolization

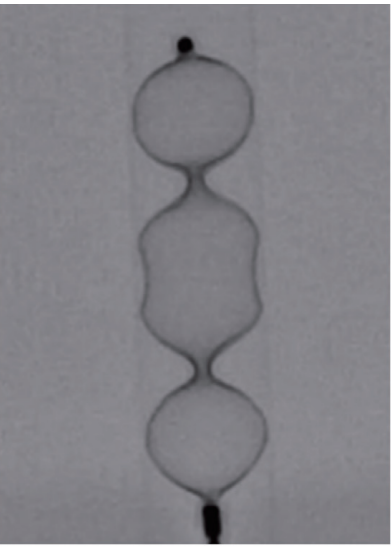

Conventional method

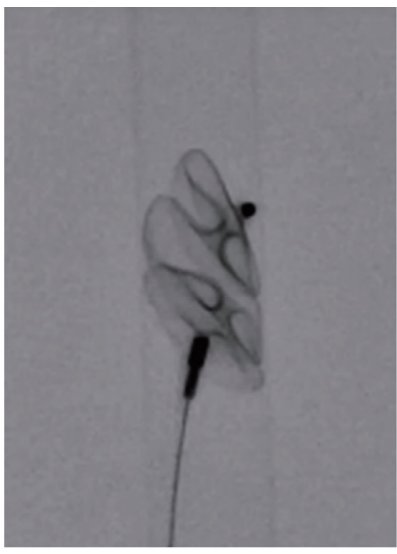

Compressing method
Fig. 2. The picture of placed AVP II by conventional and by compressing method in tube model. AVP II, Amplatzer Vascular Plug II.

Table 1. Demographics, cardiovascular risk factors, and perioperative risk assessment in 30 left subclavian artery embolizations during thoracic endovascular aortic repair

\begin{tabular}{|c|c|c|c|c|c|}
\hline & CAE group & $(n=24)$ & CCE group & $(n=6)$ & $P$ value \\
\hline Age & $76 \pm 11$ & & $79 \pm 6$ & & 0.80 \\
\hline Male gender & 22 & $91.67 \%$ & 5 & $83.33 \%$ & 0.50 \\
\hline BMI & $22.0 \pm 3.2$ & & $21.1 \pm 3.2$ & & 0.59 \\
\hline Hypertension & 21 & $87.50 \%$ & 5 & $83.33 \%$ & 1.00 \\
\hline Hyperlipidemia & 11 & $45.83 \%$ & 3 & $50.00 \%$ & 1.00 \\
\hline Coronary artery disease & 2 & $8.33 \%$ & 1 & $16.67 \%$ & 0.50 \\
\hline Prior MI & 1 & $4.17 \%$ & 0 & $0.00 \%$ & 1.00 \\
\hline $\mathrm{CHF}$ & 3 & $12.50 \%$ & 0 & $0.00 \%$ & 1.00 \\
\hline Atrial fibrillation & 1 & $4.17 \%$ & 1 & $16.67 \%$ & 1.00 \\
\hline Tobacco history & 15 & $62.50 \%$ & 5 & $83.33 \%$ & 0.63 \\
\hline COPD & 6 & $25.00 \%$ & 1 & $16.67 \%$ & 1.00 \\
\hline Diabetes mellitus & 2 & $8.33 \%$ & 1 & $16.67 \%$ & 0.50 \\
\hline CKD $(e G F R<45)$ & 10 & $41.67 \%$ & 0 & $0.00 \%$ & 0.07 \\
\hline $\mathrm{eGFR}\left(\mathrm{mL} / \mathrm{min} / 1.73 \mathrm{~m}^{3}\right)$ & $51.1 \pm 25.9$ & & $83.2 \pm 23.0$ & & 0.03 \\
\hline Cerebrovascular disease & 4 & $16.67 \%$ & 0 & $0.00 \%$ & 0.56 \\
\hline Peripheral artery disease & 2 & $8.33 \%$ & 0 & $0.00 \%$ & 1.00 \\
\hline Cancer & 7 & $29.17 \%$ & 2 & $33.33 \%$ & 1.00 \\
\hline \multicolumn{6}{|l|}{ Pathology } \\
\hline Degenerative aortic aneurysm & 21 & $87.50 \%$ & 6 & $100.00 \%$ & 1.00 \\
\hline Aortic dissection & 3 & $12.50 \%$ & 0 & $0.00 \%$ & 1.00 \\
\hline Emergency & 3 & $12.50 \%$ & 0 & $0.00 \%$ & 1.00 \\
\hline
\end{tabular}

BMI, body mass index; CAE, compressed Amplatzer Vascular Plug II embolization; CCE, conventional coil embolization; CHF, congestive heart failure; CKD, chronic kidney disease; COPD, chronic obstructive pulmonary disease; eGFR, estimated glomerular filtration rate; $\mathrm{MI}$, myocardial infarction. 


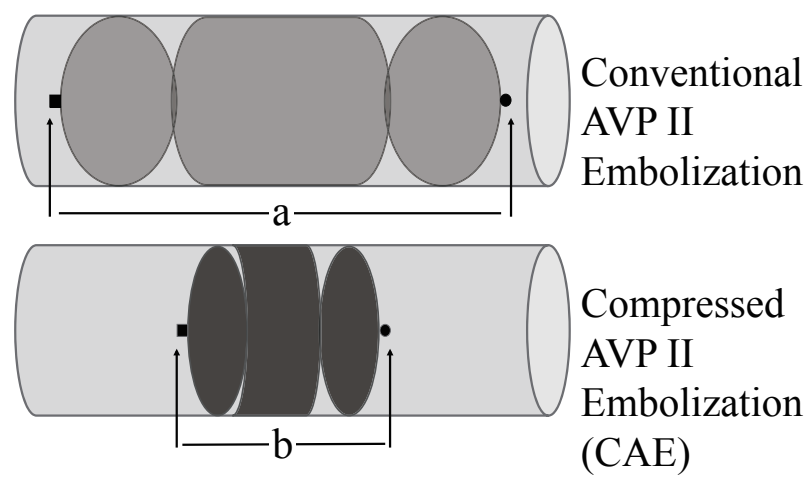

PLUG compression ratio $=\frac{a-b}{a}$

Fig. 3. The schema of conventional AVP II embolization and compressed AVP II embolization. The compression ratio of the shortening length of AVP II to the length of AVP II with conventional deployment in tube model was calculated. AVP II, Amplatzer Vascular Plug II.

ment. Consequently, we have developed an embolization technique using the compression method of all three lobes of AVP II (CAE) and confirmed the efficacy of this technique in animal experiments (unpublished data). Our aim was to evaluate safety and efficacy of CAE method during TEVAR.

\section{MATERIALS AND METHODS}

This present study was approved by the institutional review board of the authors' institution (1704A012). We retrospectively reviewed the records of 30 patients from June 2013 to March 2016. Patient demographics and pathologies are summarized in Table 1.

\section{Embolization procedures}

In the CAE group, 5 or 6-French guiding sheath introducer (Destination; Terumo Medical Corporation, Tokyo, Japan) was inserted by the transaxillary approach with surgical cut-down or percutaneous transbrachial approach. The inner diameter of the LSA was measured by contrast enhanced computed tomography (CT) in advance. The AVP II that was approximately $30-50 \%$ larger than the maximum diameter of the LSA was selected. When the AVP II reached the proximal portion of the LSA, the first lobe of the AVP II was deployed. Then, the introducer sheath was used to compress the first lobe. The second and third lobes were subsequently compressed in the same manner (Fig. 2). In the CCE group, coaxial intubation of the LSA was performed with a microcatheter (Progreat $\beta 3$; Terumo Medical Corporation, Tokyo, Japan) using a percutaneous trans-

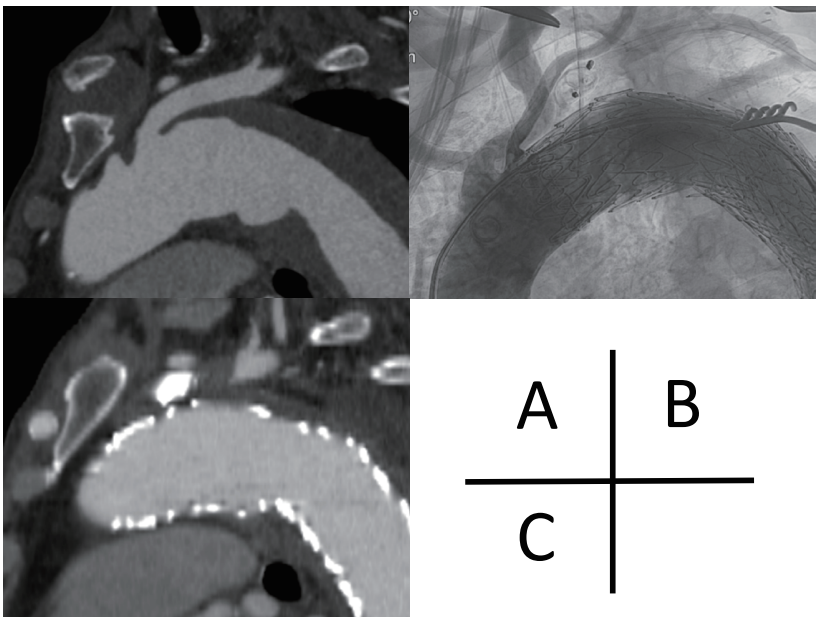

Fig. 4. Images from an 82-year-old man with type B dissection and $6.0-\mathrm{cm}$ proximal descending thoracic aneurysm treated with a stent-graft (GORE CTAG; W.L. Gore \& Associates, Flagstaff, AZ) across the origin of the subclavian artery. A: Sagittal multi-planar reconstruction image of thoracic CT angiogram before stent-grafting demonstrates the aneurysm. The proximal portion of LSA was $10-\mathrm{mm}$ in diameter and AVP II 14-mm in diameter was selected for embolization. The landing zone for AVP II embolization, from the proximal portion of LSA to the bifurcation of vertebral artery, was 42-mm long that was slightly shorter than conventional AVP II deployment in tube model 46-mm. B: Angiogram after CAE and stent-grafting. C: Sagittal multi-planar reconstruction image of thoracic $\mathrm{CT}$ angiogram after TEVAR demonstrates the absence of endoleak and that the bifurcation of vertebral artery was maintained. AVP II, Amplatzer Vascular Plug II; CAE, compressed Amplatzer Vascular Plug II embolization; CT, computed tomography; LSA, left subclavian artery; TEVAR, thoracic endovascular aortic repair.

brachial approach. LSA occlusion was achieved with detachable microcoils (Interlock; Boston Scientific, Natic, MA) with the choice of the appropriate coil size based on the diameter of the LSA. Finally, complete LSA occlusion was confirmed by retrograde digital subtraction angiography. The stent-graft deployment was done prior or subsequent to the LSA embolization.

\section{Evaluation of treatment and patient follow-up}

Technical success (defined as successful compression and placement of AVP II without sealing the LSA orifice) and complication rate of LSA embolization and the TEVAR procedure were evaluated. The amount of embolic material in the LSA by both methods was compared. To evaluate the device length of the AVP II by compression, we calculated the compression ratio by comparing the compressed AVP II length in the LSA to uncompressed AVP II length in the ex-vivo tube model (Fig. 3). For prediction of the length of deployed AVP II using the conventional technique, the length of uncompressed AVP II was measured before embolization 
in the ex-vivo tube model. When an AVP II of device size of $16 \mathrm{~mm}$ was deployed using the uncompressed conventional technique in the ex-vivo tube model whose inner diameter were 11 and $12 \mathrm{~mm}$, while the length of deployed AVP II were 52 and $49 \mathrm{~mm}$, respectively. In cases of AVP II $14 \mathrm{~mm}$ and tube models of 9, 10, and $11 \mathrm{~mm}$, the length of deployed AVP II were 50, 46, and $42 \mathrm{~mm}$, respectively. In AVP II $12 \mathrm{~mm}$ and tube models $9,8 \mathrm{~mm}$, the lengths of deployed AVP II were 36,42 mm, respectively. In AVP II $10 \mathrm{~mm}$, tube model $8 \mathrm{~mm}$, the length of deployed AVP II was $29 \mathrm{~mm}$ (unpublished data).

The costs of devices used for embolization, according to redemption prices determined in the health insurance programs in Japan, were also compared between the two groups.

The dynamic contrast enhanced CT was performed at 1 week, 6 and 12 months after the TEVAR procedure. Clinical success was defined as the absence of type II endoleak from the LSA on follow-up CT. Patient demographics, treatment modalities and outcomes were compared (Fig. 4).

A comparison of continuous variables was made using the Mann-Whitney $U$ test for independent variables, while categorical variables were compared using Fisher's exact test with the IBM SPSS Statistics version 24. Differences were considered significant if the twotailed $P$ value was $<.05$.

\section{RESULTS}

\section{Procedures and perioperative period}

The CCE was performed in 6 patients, while the CAE was performed in 24 patients. The patients in the CAE group, aged 31-90 years (mean 76.2), consisted of 22 males $(91.7 \%)$ and 2 females. The patients in the CCE group, aged 69-88 (mean 78.7), consisted of 5 males and 1 female. Both groups had similar demographics and cardiovascular risk factors, with the exception of a greater rate of chronic kidney disease in the CAE group (CAE: $42 \%$, CCE: $0 \%, P=.05$ ). Selective revascularization was performed in 3 patients $(50.0 \%)$ in the CCE group and 20 patients in the CAE group (83.3\%).

Technical success of LSA embolization was achieved at $100 \%$ in both groups (Table 2). In the CAE group, the AVP II of the device size 10, 12, 14 and $16 \mathrm{~mm}$ were used for embolization of the LSA with an inner diameter of $8-12$ (mean $10.0 \pm 0.9$ ) $\mathrm{mm}$. The length of compressed AVP II was 15-24 (mean $19.0 \pm$ 3.6) $\mathrm{mm}$. The length of the proximal portion of the LSA from the LSA orifice to the vertebral artery origin was 24-62 (mean $41.7 \pm 8.9$ ) $\mathrm{mm}$ in the CAE group and the length of uncompressed AVP II was 29-52 (mean 45.9 $\pm 4.9)$ in the tube model. In seventeen patients of the CAE group (70.8\%), the length of uncompressed AVP II in the tube model was equal to or larger than that of the proximal portion of the LSA from the LSA orifice to the vertebral artery origin in the CAE group. The mean compression ratio of AVP II was $58.6 \pm 5.9 \%$.

Table 2. Left subclavian artery embolization details

\begin{tabular}{|c|c|c|c|}
\hline & $\begin{array}{l}\text { CAE group } \\
(n=24)\end{array}$ & $\begin{array}{l}\text { CCE group } \\
(n=6)\end{array}$ & $P$ value \\
\hline Technical success & $24(100 \%)$ & $6(100 \%)$ & 1.00 \\
\hline $\begin{array}{l}\text { Diameter of LSA proximal } \\
\text { portion }(\mathrm{mm})\end{array}$ & $10.0 \pm 1.1$ & $9.5 \pm 1.5$ & 0.47 \\
\hline Landing zone in LSA (mm) & $41.7 \pm 8.9$ & $42.7 \pm 6.3$ & 0.98 \\
\hline $\begin{array}{l}\text { The length of embolization } \\
\text { material }(\mathrm{mm})\end{array}$ & $19.0 \pm 3.6$ & $33.2 \pm 5.8$ & $* *<0.01$ \\
\hline \multicolumn{4}{|c|}{$\begin{array}{l}\text { The length of the uncom- } \\
\text { pressed AVP II with standard } 45.9 \pm 4.9 \\
\text { procedure in tube model (mm) }\end{array}$} \\
\hline No. of embolization materials & 1 & $10.2 \pm 2.7$ & $* *<0.01$ \\
\hline $\begin{array}{l}\text { Cost of embolization materi- } \\
\text { als (JPY) }\end{array}$ & 129,000 & $\begin{array}{l}639,600 \\
\pm 140,060\end{array}$ & $* *<0.01$ \\
\hline
\end{tabular}

AVP II, Amplatzer Vascular Plug II; CAE, compressed Amplatzer Vascular Plug II embolization; CCE, conventional coil embolization; JPY, Japanese yen; LSA, Left subclavian artery.

In the CAE group, emergency TEVAR was applied for ruptured thoracic aortic aneurysm. No major complication such as a stroke or shower embolism was noticed in all but one patient. One patient died from a re-rupture of an aneurysmal sac due to type $1 \mathrm{~b}$ endoleak seven days after the TEVAR. Although a larger-sized introducer was used in CAE, there was no significant difference in any access route-related complications in both groups.

The number of embolization material in the CAE group and CCE group were one and $10.2 \pm 2.7$, respectively $(P<.01)$. The costs incurred from embolization material were lower in the CAE group (CAE: 129,000 JPY, CCE: 639,600 \pm 140,060 JPY, $P<.01$ ).

\section{Follow-up period}

The mean follow-up period was 381 days (range, 7-768 days) in the CAE group, and 942 days (range, 714-1116 days) in the CCE group. Although type II endoleak was detected in five CAEs and three CCEs, no LSA-related endoleak was observed. Migration of any embolic material was not found. No ischemic symptoms of the left arm due to the LSA embolization were recorded throughout the follow-up period. Aneurysmal sac enlargement of more than $5 \mathrm{~mm}$ in diameter was not observed in any case within the follow-up period. 


\section{DISCUSSION}

TEVAR has become increasingly popular in the treatment of aneurysms and type B aortic dissections. ${ }^{13-17}$ When the proximal end of the aneurysmal sac or entry tear is close to the orifice of LSA, it is often necessary to cover the LSA orifice to establish this required landing zone for stent-grafting. Patency of the proximal portion of the LSA may cause a type II endoleak. Type II endoleaks may resolve spontaneously, but persistent pressurization of the aneurysmal sac places the patient at risk of sac rupture. ${ }^{5}$ Consequently, the presence of a type II endoleak, particularly in the setting of aneurysm sac enlargement, is an indicator of a poor clinical outcome and requires repair. ${ }^{9}$ LSA occlusion, with or without surgical arch debranching, is needed to prevent type II endoleak. Conventional occlusion methods of the proximal portion of the LSA to deal with type II endoleak include surgical ligation or catheter-directed embolization with metal coils or glue. ${ }^{3}$

AVP II prosthesis, which is an excellent vessel occlusion device for medium to large vessels with high flow, consists of nitinol mesh with varying numbers of wires (144 to 360) with different designs (one to three layers of wires). It has three lobes of equal diameter without the presence of tissue inside. Meyer et al. reported AVP II to be a safe and easy-to-use instrument demonstrating rapid permanent occlusion of the left-subclavian back flow type II endoleak. ${ }^{18}$ However, AVP II embolization requires a specific length of arterial segment because three lobes of AVP II are deployed by their extension in the arterial lumen. In addition, the length of the proximal portion of the LSA from the LSA orifice to the vertebral artery origin of the LSA is often too short for AVP II placement with the uncompressed conventional technique. In our study, the length of the proximal potion of the LSA was equal to or shorter than AVP II length with the conventional procedure in the tube model in seventeen patients of the CAE group (70.8\%). Even in such situations, we could successfully embolize the proximal portion of the LSA using compressed AVP II.

Concerning the application of compressed AVP II for the embolization of LSA, there are several risks to consider. The lobe deformity of AVP II by compression might cause insufficient occlusion. In addition, the compression may cause distal embolization from atherothrombotic debris because of abrasion of the surface of the artery from the mesh lobe during the procedure. To resolve these risks, we have performed animal experiments using swine (unpublished data). From this study, we have histologically confirmed the safety of compression of AVP II. In all CAE patients of our series, AVP IIs were adapted to LSA embolization by compression.
CAE could provide effective LSA embolization without any complications on TEVAR combined with or without debranching bypass. Although, a larger-sized introducer sheath is necessary to deliver the AVP II, no significant differences in complication rate were observed between CAE and CCE.

The cost of embolization devices such as Amplatzer vascular plugs and standard coils have been reviewed by several investigators. American investigators found that the Amplatzer vascular plug was 69\% less expensive based on the cost of the coils or plug alone in transluminal hypogastric artery occlusion during abdominal endovascular aortic repair. ${ }^{17}$ In the series by Vandy et al. ${ }^{17} 7.53$ coils were utilized compared with 1.35 plugs. European investigators found that the Amplatzer plug was $71 \%$ less expensive based on the cost of the devices alone for occlusion of the internal iliac artery. ${ }^{19}$ In our series, 10.2 coils were placed compared with only one plug. As such, the CAE cost $80 \%$ less than the CCE, corroborating findings from those reports. As such, CAE can reduce the cost of embolization devices.

Being a retrospective study, the present study has several limitations including lack of randomization. In addition, the sample size is relatively small, especially in the CCE group. First, although no major stroke was seen in this study, we did not evaluate the incidence of subclinical cerebral infarction during the embolization procedure. Further examination is required with regard to the incidence of micro cerebral embolism. Second, the procedure time of CCE or CAE could not be compared because it was not recorded, while the whole procedure time of TEVAR was recorded. Third, the pressure on the vessel wall by compressing method was not evaluated.

In the present study, we retrospectively reviewed success rate and safety of CAE and compared with CCE. We designed the CAE for prevention of type II endoleaks associated with TEVAR for distal arch pathology. CAE was found to be safe and effective for LSA proximal embolization. CAE can reduce the cost of embolization devices and as such, CAE could be a candidate to supplant CCE.

The authors declare no conflict of interest.

\section{REFERENCES}

1 Peterson MD, Wheatley GH, 3rd, Kpodonu J, Williams JP, Ramaiah VG, Rodriguez-Lopez JA, et al. Treatment of type II endoleaks associated with left subclavian artery coverage during thoracic aortic stent grafting. J Thorac Cardiovasc Surg. 2008;136:1193-9. PMID: 19026802.

2 Kotelis D, Geisbusch P, Hinz U, Hyhlik-Durr A, von TenggKobligk H, Allenberg JR, et al. Short and midterm results after left subclavian artery coverage during endovascular repair of the thoracic aorta. J Vasc Surg. 2009;50:1285-92. PMID: 
19837529.

3 Sunder-Plassmann L, Orend KH. Stentgrafting of the thoracic aorta-complications. J Cardiovasc Surg (Torino). 2005;46:12130. PMID: 15793491.

4 Woo EY, Carpenter JP, Jackson BM, Pochettino A, Bavaria JE, Szeto WY, et al. Left subclavian artery coverage during thoracic endovascular aortic repair: a single-center experience. J Vasc Surg. 2008;48:555-60. PMID: 18572361.

5 Tholpady A, Hendricks DE, Bozlar U, Turba UC, Sabri SS, Angle JF, et al. Percutaneous occlusion of the left subclavian and celiac arteries before or during endograft repair of thoracic and thoracoabdominal aortic aneurysms with detachable nitinol vascular plugs. J Vasc Interv Radiol. 2010;21:1501-7. PMID: 20801685.

6 Parmer SS, Carpenter JP, Stavropoulos SW, Fairman RM, Pochettino A, Woo EY, et al. Endoleaks after endovascular repair of thoracic aortic aneurysms. J Vasc Surg. 2006;44:44752. PMID: 16950414.

7 Parodi JC. Endovascular repair of abdominal aortic aneurysms and other arterial lesions. J Vasc Surg. 1995;21:549-55; discussion 56-7. PMID: 7707560.

8 Lumsden AB, Allen RC, Chaikof EL, Resnikoff M, Moritz MW, Gerhard H, et al. Delayed rupture of aortic aneurysms following endovascular stent grafting. Am J Surg. 1995;170:174-8. PMID: 7631925.

9 Czerny M, Grimm M, Zimpfer D, Rodler S, Gottardi R, Hutschala D, et al. Results after endovascular stent graft placement in atherosclerotic aneurysms involving the descending aorta. Ann Thorac Surg. 2007;83:450-5. PMID: 17257968.

10 Ryer EJ, Garvin RP, Webb TP, Franklin DP, Elmore JR. Comparison of outcomes with coils versus vascular plug embolization of the internal iliac artery for endovascular aortoiliac aneurysm repair. J Vasc Surg. 2012;56:1239-45. PMID: 22727840.

11 Ratnam LA, Walkden RM, Munneke GJ, Morgan RA, Belli AM. The Amplatzer vascular plug for large vessel occlusion in the endovascular management of aneurysms. Eur Radiol. 2008;18:2006-12. PMID: 18418603.

12 Iida Y, Ito T, Hayashi S, Takahashi T, Misumi T, Hachiya T, et al. Repair of Acute Type B Aortic Dissection Complicated by Aortic Rupture with Debranching Thoracic Endovascular Aortic Repair and Left Subclavian Artery Occlusion Using Amplatzer Vascular Plug II. Ann Vasc Dis. 2015;8:252-4. PMID: 26421077.

13 Chung J, Kasirajan K, Veeraswamy RK, Dodson TF, Salam AA, Chaikof EL, et al. Left subclavian artery coverage during thoracic endovascular aortic repair and risk of perioperative stroke or death. J Vasc Surg. 2011;54:979-84. PMID: 21658894.

14 Chung J, Corriere MA, Veeraswamy RK, Kasirajan K, Milner R, Dodson TF, et al. Risk factors for late mortality after endovascular repair of the thoracic aorta. J Vasc Surg. 2010;52:549-54;discussion 55. PMID: 20598483.

15 Matsumura JS, Lee WA, Mitchell RS, Farber MA, Murad MH, Lumsden AB, et al. The Society for Vascular Surgery Practice Guidelines: management of the left subclavian artery with thoracic endovascular aortic repair. J Vasc Surg. 2009;50:1155-8. PMID: 19878791.

16 Baba T, Ohki T, Kanaoka Y, Maeda K. Clinical Outcomes of Left Subclavian Artery Coverage on Morbidity and Mortality During Thoracic Endovascular Aortic Repair for Distal Arch Aneurysms. World J Surg. 2015;39:2812-22. PMID: 26220365.

17 Vandy F, Criado E, Upchurch GR, Jr., Williams DM, Rectenwald J, Eliason J. Transluminal hypogastric artery occlusion with an Amplatzer vascular plug during endovascular aortic aneurysm repair. J Vasc Surg. 2008;48:1121-4. PMID: 18692350.

18 Meyer C, Probst C, Strunk H, Schiller W, Wilhelm K. Second-generation Amplatzer Vascular Plug (AVP) for the treatment of subsequent subclavian backflow type II endoleak after TEVAR. Cardiovasc Intervent Radiol. 2009;32:1264-7. PMID: 19280256.

19 Pellerin O, Caruba T, Kandounakis Y, Novelli L, Pineau J, Prognon P, et al. Embolization of the internal iliac artery: cost-effectiveness of two different techniques. Cardiovasc Intervent Radiol. 2008;31:1088-93. PMID: 18612682. 